\title{
Neuronal congruency effects in macaque frontal cortex
}

Tao Yao ( $\nabla$ taoyao12@hotmail.com )

KU Leuven https://orcid.org/0000-0003-2060-690X

\section{Wim Vanduffel}

KU Leuven

\section{Article}

Keywords: behavioral congruency effect, neuronal mechanisms, selective attention

Posted Date: November 19th, 2021

DOI: https://doi.org/10.21203/rs.3.rs-1065497/v1

License: (9) This work is licensed under a Creative Commons Attribution 4.0 International License. Read Full License

Version of Record: A version of this preprint was published at Nature Communications on August 10th, 2022. See the published version at https://doi.org/10.1038/s41467-022-32382-1. 


\section{Affiliations:}

${ }^{1}$ Department of Neurosciences, Laboratory of Neuro- and Psychophysiology, KU Leuven Medical School, Leuven 3000, Belgium

${ }^{3}$ Athinoula A. Martinos Center for Biomedical Imaging, Massachusetts General Hospital, 9 Charlestown, MA 02129, USA

*Correspondence to:

13 Wim Vanduffel, WVanduffel@mgh.harvard.edu; Tao Yao, tao.yao@kuleuven.be 
The interplay between task-relevant and task-irrelevant stimulus features induces conflicts which impair human behavioral performance in many perceptual and cognitive tasks, a.k.a. a behavioral congruency effect. The neuronal mechanisms underlying behavioral congruency effects, however, are poorly understood. We recorded single unit activity in monkey frontal cortex using a novel task-switching paradigm and discovered a neuronal congruency effect that is carried by task-relevant and -irrelevant neurons. The former neurons provide more signal, the latter less noise in congruent compared to incongruent conditions. Their relative activity levels determine the neuronal congruency effect and behavioral performance. Although these neuronal congruency signals are sensitive to selective attention, they cannot be entirely explained by selective attention as gauged by response time. We propose that such neuronal congruency effects can explain behavioral congruency effects in general, as well as previous fMRI and EEG results in various conflict paradigms. 
Cognitive control is essential for flexible goal-directed behavior allowing us to quickly adapt to continuously changing environments and contexts. A proposed cognitive control mechanism is that, depending on internal goals or external context, subjects allocate attention to task-relevant information while they ignore task-irrelevant information to optimize behavioral performance ${ }^{1-3}$. Sometimes, however, task-irrelevant information has also to be processed to some degree, which can interfere with task (relevant) performance. The cocktail party is a classic situation whereby perception of your interlocutor's voice is typically not dominated by surrounding sounds. Yet in some instances surround information might interfere with your conversation, for example when your name is called by a remote individual, not directly participating in the conversation ${ }^{4}$. In laboratory settings, interference from task-irrelevant information is frequently investigated using 'conflict tasks', including the Stroop, Flanker, Simon and Wisconsin card sorting task, but also pro/anti-saccade, and countermanding tasks ${ }^{5-11}$. In high conflict, or incongruent conditions, errors and reaction times increase compared to low conflict or congruent conditions, a behavioral phenomenon known as the congruency effect ${ }^{12,13}$. For example, in incongruent trials of the Stroop task, the meaning of a word interferes with the congruent trials (e.g. "RED” printed in red color).

Previous imaging and electrophysiological studies in humans and non-human primates revealed fronto-parietal areas as important players in conflict processing ${ }^{5,13-28}$. However, most of these studies focused on conflict detection and resolution, not on the neuronal mechanisms underlying the behavioral congruency effect: “...what makes an incongruent trial slower or more 
1 error-prone..."29. We aimed to investigate the neuronal implementation of behavioral congruency effects, i.e., the neuronal congruency effect, which is the foundation of multiple (sometimes contradictory) theories and models ${ }^{12,16,30-33}$. To this end, we recorded single unit activity in the monkey's frontal eye fields (FEF) while the subjects were performing a taskswitching paradigm (Figure 1). Across congruent and incongruent trials, we controlled the visual input, the motor output, and the allocation of voluntary spatial attention. The FEF contains a topographic map of visual saliency, whereby target locations are represented by the peak activity within $\mathrm{FEF}^{34}$. Therefore, FEF is an ideal area to investigate differences in target selection between congruent and incongruent trials ${ }^{35}$.

Hypothetically, since two stimuli (target and distractor) were presented in our task, two major neuron populations may affect target selection within the FEF: task-relevant neurons (with targets inside their receptive field (RF)), and task-irrelevant neurons (with distractors inside their RFs) (Figure 1A, bottom). The activity of task-relevant and task-irrelevant neurons, referred to as 'signal' and 'noise' for target selection in this context, would have a positive and negative effect on target selection, respectively. We predict that the signal-to-noise ratio (SNR) within FEF's saliency map is lower in incongruent than congruent conditions, leading to a behavioral congruency effect (Figure 1A, top) in our task. Moreover, there are three possible scenarios to achieve lower SNR in incongruent trials (Figure 1A, bottom): decreased signal (left), increased noise (middle), and decreased signal \& increased noise (right). Our first goal was to determine the activity in task-relevant and -irrelevant neurons with our paradigm, and which of the three 

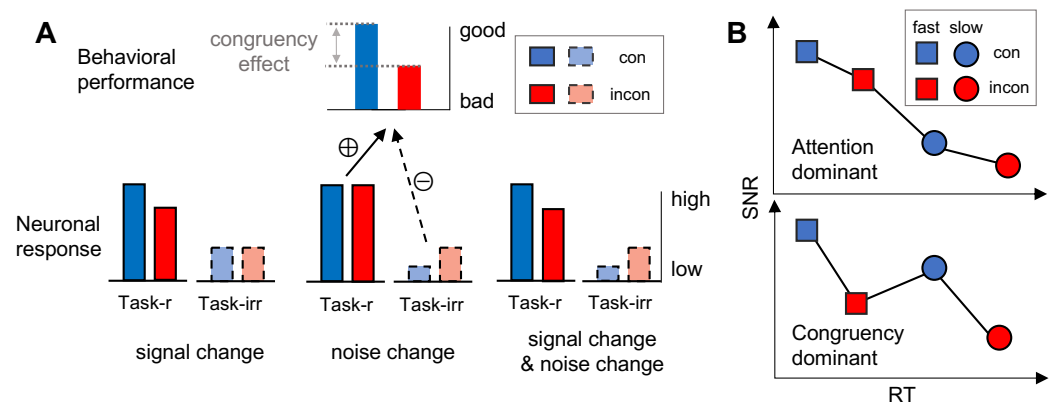
congruency effects. A. The top represents the behavioral congruency effect, i.e., worse performance in incongruent than congruent conditions. In brain areas closely related to the output, at least three possible scenarios can lead to behavioral congruency effects by changing the response amplitudes of task-relevant (signal) and/or task-irrelevant (noise) neurons. The signal to noise ratio (SNR) represents how well a target is represented in FEF and can be selected by the subject. All three possibilities (bottom plots) lead to lower SNR in incongruent conditions. Left: task-relevant neurons show lower response in incongruent condition while activity in task-irrelevant neurons remains unchanged. Middle: task-irrelevant neurons show higher response in incongruent condition, while task-relevant neurons are unaffected. Right: task-relevant and-irrelevant neurons show lower and higher activity in incongruent condition, respectively.

B. Two possible mechanisms explaining SNR in our task. Top: SNR is dominated by attention (indexed by RT, with shorter RTs implying that attention was more focused on the target). Bottom: SNR is dominated by congruency. 


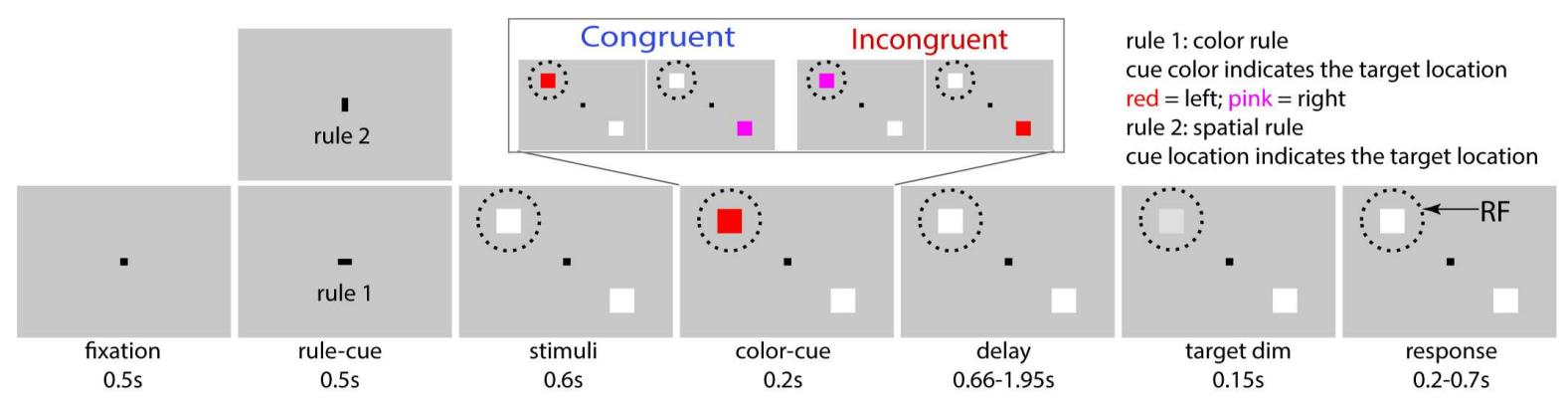

Figure 2. The task paradigm. Subjects were required to pay covert attention to a target stimulus and respond to its dimming. Subjects initiated trials by foveating a fixation point (FP) which turned to a horizontal or vertical bar (task rule-cue: color or spatial rule). Then, the FP reappeared simultaneously with two peripheral white squares. Next, one square turned to pink or red, serving as color-cue. In trials with horizontal bars, the color of the color-cue determined target location (red and pink indicating a target on the left and right, respectively), its spatial location being irrelevant. Conversely (vertical bar), the location of the color-cue indicated the position of the target, its color being irrelevant. Trials were subdivided into congruent (i.e., red presented on the left, and pink on right) and incongruent conditions (vice versa) based on the spatial location and color of the color-cue. Monkeys had to respond to target dimming by pressing a button with their left hand to obtain a reward. To ensure the monkeys paid attention to the target only, monkeys had to ignore distractor dimming, occurring in $50 \%$ of the trials (before target dimming). All trial types were pseudo-randomly interleaved. The (virtual) dashed circle indicates the neuron's RF. The monkeys had to foveate to the FP during the entire trial.

Selective attention is a key component required during all conflict tasks $2,29,36,37$. Selective spatial or featural attention can also lead to enhanced and suppressed activity in task-relevant and -irrelevant neurons, respectively ${ }^{38,39}$. Hence selective attention can also change the SNR within 
FEF. A second question is whether we can, at least partially, dissociate attentional from congruency mechanisms underlying the behavioral congruency effect. Response times (RTs) are faster when subjects are more certain about the location of a target or when attention is more focused on the target ${ }^{40-44}$. To test the selective attention hypothesis, we compared the SNR of FEF neurons between trials with fast and slow RTs, indicative for high and low levels of selective attention/certainty, respectively. If the SNR differences between congruent and incongruent conditions are dominated by selective attention as indicated by RT, we expect a negative correlation between RT and SNR (Figure 1B top). On the other hand, if the SNR is dominated by congruency in our experiment, we expect higher SNR for congruent than incongruent conditions, independent of RT (Figure 1B bottom).

\section{RESULTS}

\section{Behavioral congruency effects with the task-switching paradigm}

Two rhesus monkeys (Macaca mulatta) were trained to perform the task-switching paradigm (Figure 2, methods). Monkeys were required to covertly attend to a target and detect its dimming by pressing a button while ignoring distractor stimuli. Target locations were determined by a combination of a task rule-cue (color or spatial rule), and a color-cue (pink or red). A trial was considered congruent when the color and spatial location of the color-cue indicated the same target location under both rules (Table S1). After extensive training and before the electrophysiological recordings started, performance levels remained stable. During 29 recording sessions (12 in Monkey S, 17 in Monkey R), we found significant behavioral congruency effects for both task rules, consistently across left and right target locations (Figure 3 and Table S2). The average performance was higher for congruent than incongruent trials 
(monkey S: $93.8 \%$ vs. $80.6 \%, t_{11}=16.07, p=5.5 e-9$; monkey R: $95.9 \%$ vs. $81.6 \%, t_{16}=13.5$, $p=3.7 e-10)$.

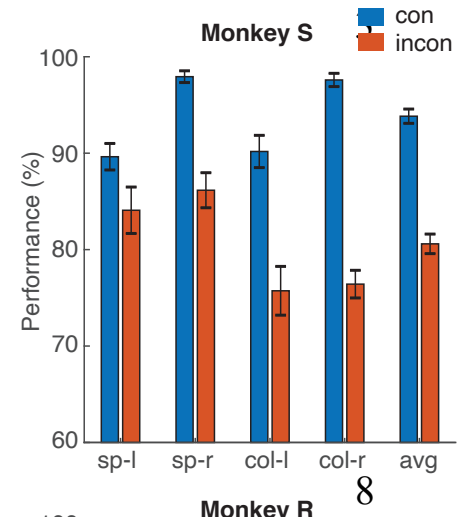

Figure 3. Behavioral congruency effect. The performance (proportion correctly identified target dimming) for congruent (blue) and incongruent (red) trials during the electrophysiological recording sessions. A behavioral congruency effect was found for both rules and all target locations. All pairwise comparisons between congruent and

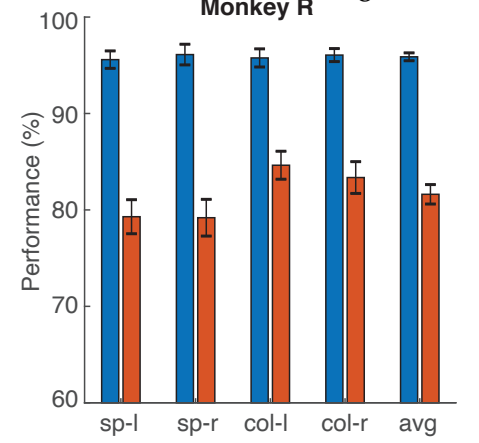
incongruent conditions were significant (two tailed paired ttest). Error bars: SEM across sessions. sp: spatial rule; col: color rule; l: left target; r: right target; avg: average performance across conditions.

\section{Both task-relevant and -irrelevant FEF neurons are modulated by congruency}

To study neuronal correlates of the behavioral congruency effect, we recorded single units with 16-channel probes from the right FEF (Figure S1) in two monkeys. In total, 248 visual neurons $(121 / 127$ from monkey S/R) with contralateral RFs were included in our analysis (Methods). All trials were categorized into four conditions based on conflict level (congruent or incongruent) and target locations relative to the RFs (TarIn or TarOut). Since we are most interested in the neuronal representations of the behavioral congruency effect, and not necessarily conflict detection and resolution, we focused our analysis on a $500 \mathrm{~ms}$ time window immediately before the target dimmed. This is the period closest to the behavioral response, when the conflict should have been detected and solved given the long delay. As expected and 
consistent with previous studies ${ }^{45-47}$, FEF neurons were significantly modulated by covert spatial attention: average responses to congruent trials were higher for targets inside (solid blue lines) compared to outside (dashed blue lines) the neurons' RF (Figure 4A, 4B). Attention increased the neuronal responses by a median value of $50 \%$ in monkey S (Wilcoxon Signed Rank Test, WSRT, $\mathrm{p}=8.2 \mathrm{e}-13$ ) and $22.2 \%$ in monkey R (WSRT, $\mathrm{p}=4 \mathrm{e}-11$ ), which also reassured that the target stimuli were inside the neuron's RFs.

Critically, our data indicate that behavioral congruency effects can be explained by changing responses of both task-relevant (representing signal power) and task-irrelevant (representing noise power) neurons (Figure 1A). Specifically, responses of task-relevant neurons (TarIn) were lower for incongruent (solid blue lines, Figure 4A and 4B) compared to congruent conditions (solid orange lines), corresponding to a decrease in signal to target selection in $=6.4 \mathrm{e}-5)$, indicating that more task-relevant neurons responded higher in congruent than incongruent conditions. 107 (43\%) neurons showed significantly different responses between congruent and incongruent conditions (dark bars in Figure 4C and 4E), from which 81 neurons 
1 significantly higher in congruent versus incongruent trials. Only 26 neurons (24\%; i.e., 12 (24\%) and $14(25 \%)$ for S and R) showed the opposite effect.
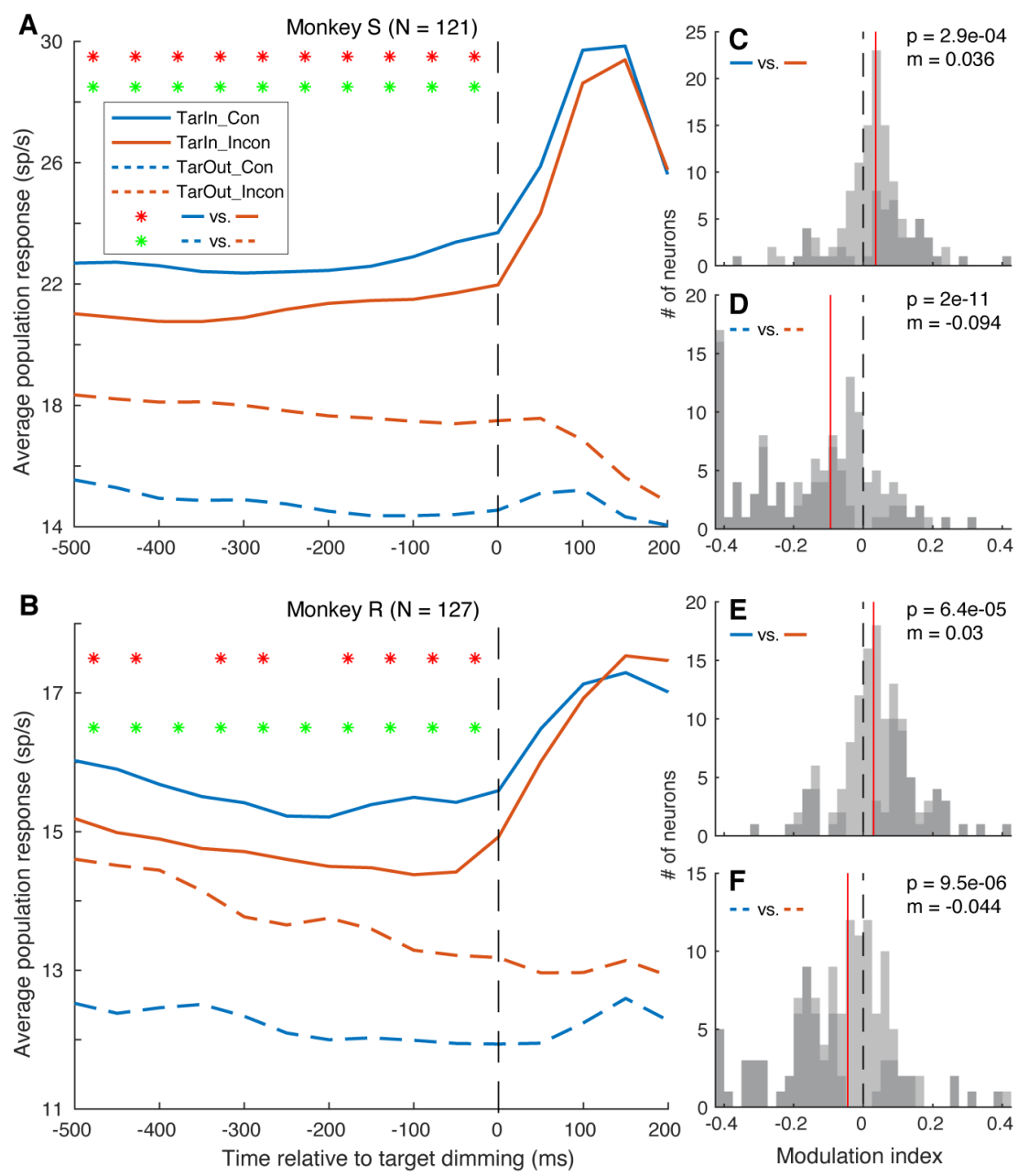

Figure 4. Neuronal congruency effect in $\mathbf{F E F}$. $(\boldsymbol{A}, \boldsymbol{B})$ The population average peristimulus time histograms aligned to target dimming (vertical dashed line) indicate a signal decrease (difference between solid blue and orange lines) when targets fall inside the RF (TarIn), and a noise increase (difference between dashed blue and orange lines) when targets are presented outside the RF (TarOut) in incongruent compared to incongruent conditions. Blue and orange lines represent congruent (Con) and incongruent (Incon) conditions, respectively. Red stars indicate significantly different responses (congruent versus incongruent) for 
successive, non-overlapping $50 \mathrm{~ms}$ bins for TarIn (i.e., significant signal change, two tailed paired t-test, $p<0.05$ ). Green stars indicate significantly different responses for TarOut (i.e., significant noise change). (C, E) Frequency histograms of modulation indices $\left(M I=\left(R_{\text {con }}{ }^{-}\right.\right.$ $\left.\left.R_{\text {incon }}\right) /\left(R_{\text {con }}+R_{\text {incon }}\right)\right)$, $R$ : average response 500ms prior target dimming) in monkey $S(C)$ and monkey $R(\boldsymbol{E})$ for TarIn. MI values $>0$ : signal increase in congruent than incongruent trials. The p-value (Wilcoxon signed rank test, WSRT) and median MI values (m) are indicated. Black vertical dashed lines indicate no signal change; red lines the median MI. All values $>0.4$ or $<-$ 0.4 are summed in the last and first data point of each histogram, respectively. Dark bars indicate neurons showing significantly different responses (two tailed paired t-test, $p<0.05$ ) between congruent and incongruent conditions.

$(\boldsymbol{D}, \boldsymbol{F})$ Same as

C, $\boldsymbol{E}$ but for TarOut. The distribution of MIs in $\boldsymbol{D}$ and $\boldsymbol{F}$ shows a predominance of values smaller than zero: noise

On the other hand, the response of task-irrelevant neurons (TarOut) immediately before target dimming was higher, reflecting increased noise for incongruent (dashed orange lines) compared to congruent conditions (dashed blue lines). This increased noise in task-irrelevant neurons was consistent in both monkeys, and significant for every $50 \mathrm{~ms}$ bin $500 \mathrm{~ms}$ prior target dimming (green stars, two tailed paired t-test, $\mathrm{p}<0.05$ ). The distribution of MIs (Figure 4D and 4F) shifted towards negative values (WSRT, S: median $=-0.094, p=2 \mathrm{e}-11$; R: median $=-0.044$, $\mathrm{p}=9.5 \mathrm{e}-6)$, indicating that more neurons responded higher in incongruent than congruent trials. 142 neurons (57\%) showed significantly different responses between congruent and incongruent trials (dark bars in Figure 4D and 4F), from which 116 neurons (82\%; i.e., $66(86 \%)$ and 50 26 neurons (18\%; i.e., 11 (14\%) and 15 (23\%) for S and R) showed the opposite effect. 
In sum, our results indicate that the neuronal congruency effect is a combination of opposite response changes in task-relevant and -irrelevant neurons, resulting in an SNR decrease to target selection within FEF's saliency map in incongruent compared to congruent conditions, confirming the third option in Figure 1A.

Both spatial location and symbolic meaning of the color-cues interfere with target selection within FEF

The behavioral performance of the subjects was lower in incongruent compared to congruent conditions independent of the spatial or color rule, indicating that both the location and symbolic meaning of stimuli interfered with performance in incongruent trials. Hence an important question is whether both spatial and symbolic features modulate the neuronal responses in FEF in a congruency-dependent manner. In color-rule trials, subjects had to identify the color and translate it into a spatial location to shift their attention accordingly (i.e., they had to understand the symbolic meaning of the color). This is a more complex task and potentially involves different pathways compared to the spatial processing required during the spatial-rule trials. The latter trials might rely on the dorsal visual pathway, while color-rule trials might depend more on the ventral stream ${ }^{48}$. Therefore, the neuronal congruency effects under these two rules might be very different in FEF. We analyzed the signal and noise changes separately for both rules during a 500ms interval before target dimming, exactly as in Figure 4. Compared to congruent trials, both rules led to response decreases in task-relevant neurons (signal decreases) (Monkey S: Figure 5A, 5C and Figure S3, monkey R: Figure 5E and 5G) and response increases in task-irrelevant neurons (noise increases) during incongruent conditions (Monkey S: Figure 5B and 5D, Monkey R: Figure 5F and 5H). Hence, basic as well as complex features (e.g., the 
1 spatial location and symbolic meaning of cues in our task) can induce behavioral and neuronal congruency effects.

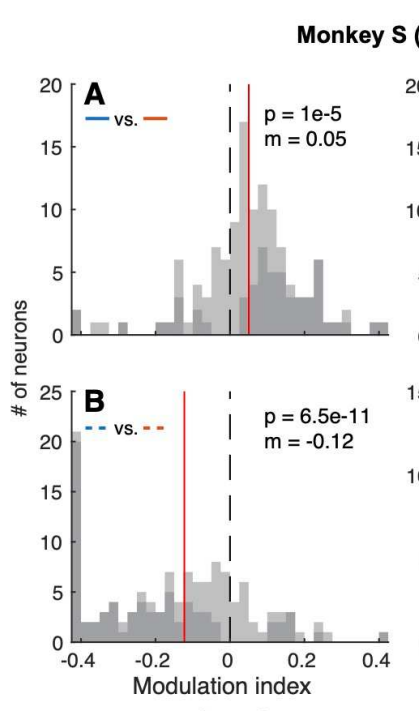

Monkey $S(N=121)$
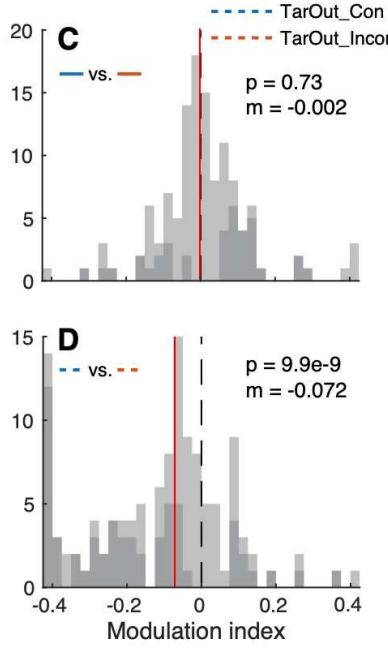

spatial rule

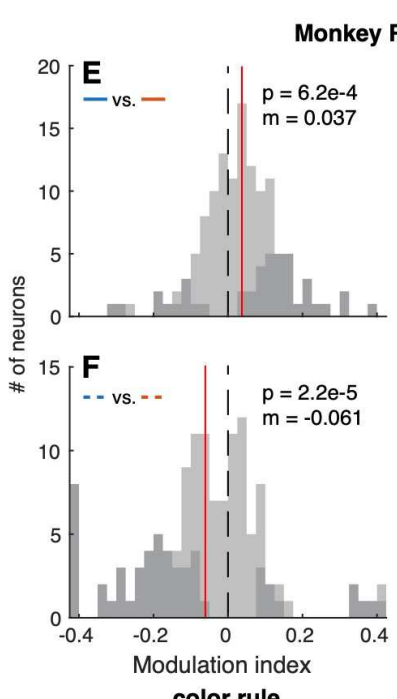

color rule
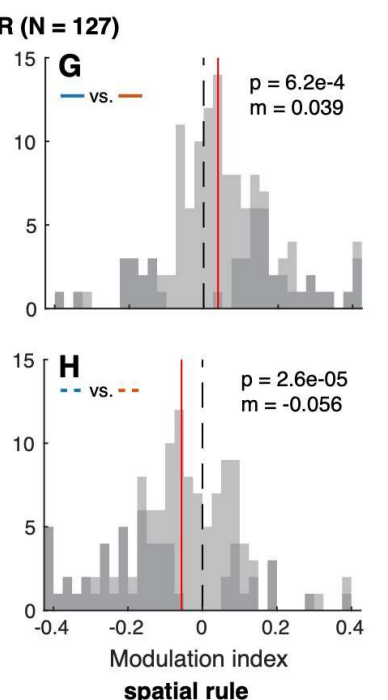

19

Figure 5. Neuronal congruency effects under color- and spatial-rules. The neuronal congruency effects are present under both rule tasks in monkey $S(\boldsymbol{A}-\boldsymbol{D})$ and monkey $R(\boldsymbol{E}-\boldsymbol{H})$. The conventions are the same as in Figure $3 C-F$, but the color-rule task (left column, $\boldsymbol{A}, \boldsymbol{B}, \boldsymbol{E}, \boldsymbol{F}$ ) and the spatial-rule task (right column, $\boldsymbol{C}, \boldsymbol{D}, \boldsymbol{G}$, H) are separated. A, C, E, G are showing the signal change between congruent and incongruent trials, and $\boldsymbol{B}, \boldsymbol{D}, \boldsymbol{F}, \boldsymbol{H}$ are showing the noise change. 

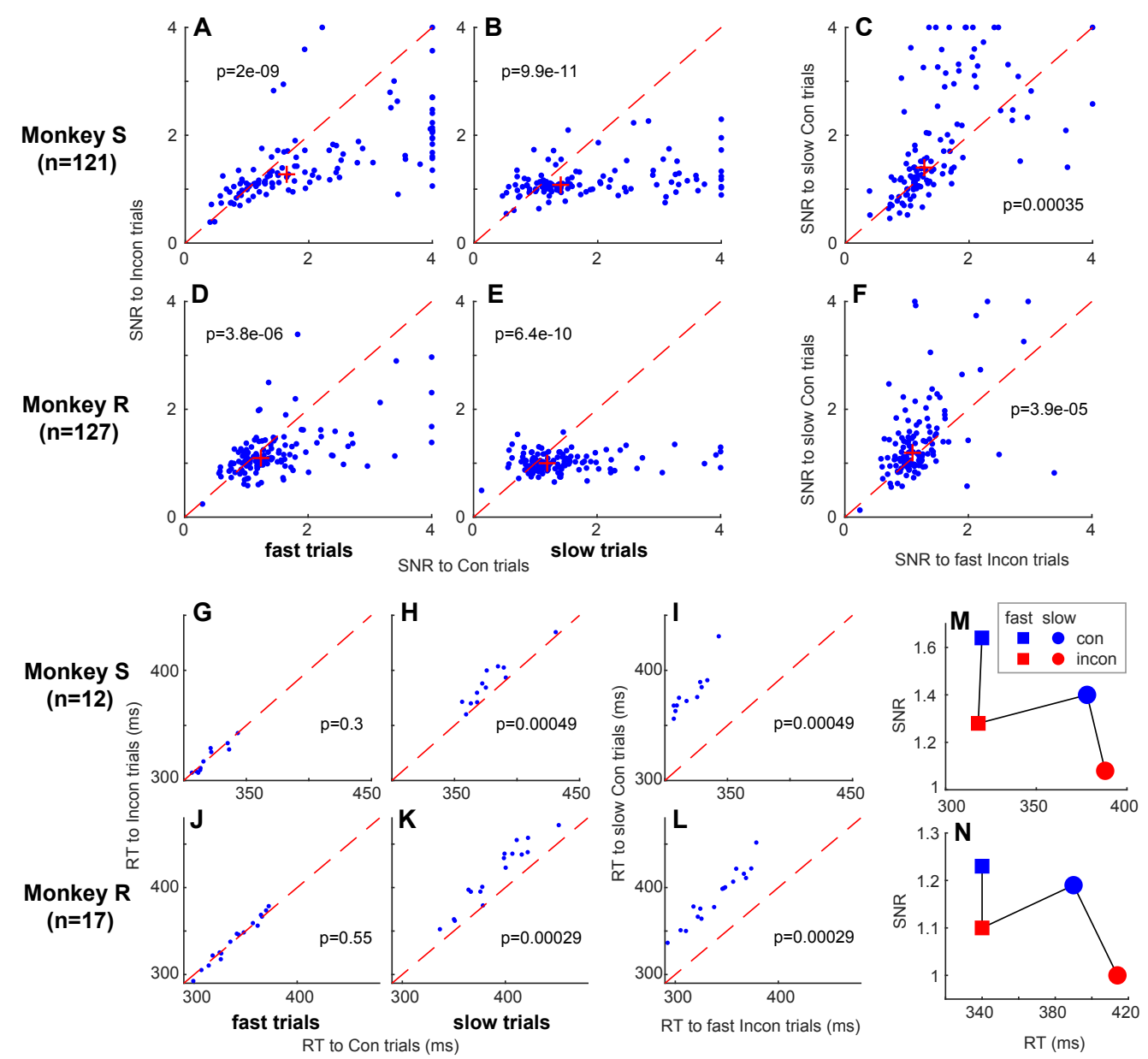

Figure 6. The SNR within FEF and RT related to congruency effect. The $S N R(\boldsymbol{A}, \boldsymbol{D})$ and $R T$ $(\boldsymbol{G}, \boldsymbol{J})$ of congruent and incongruent conditions for fast trials. The $\operatorname{SNR}(\boldsymbol{B}, \boldsymbol{E})$ and $R T(\boldsymbol{H}, \boldsymbol{K})$ of congruent and incongruent conditions for slow trials. The SNR $(\boldsymbol{C}, \boldsymbol{F})$ and $R T(\boldsymbol{I}, \boldsymbol{L})$ of fast incongruent and slow congruent trials. SNR values higher than 4 are plotted as 4. X and Y labels indicate the conditions being compared. Each dot in $\boldsymbol{A}-\boldsymbol{F}$ and $\boldsymbol{G}-\boldsymbol{L}$ represents a neuron and a recording session respectively. $S N R=R_{\text {TarIn }} / R_{\text {TarOut }}, R$ : average response $500 m s$ prior target dimming for the conditions compared. The red dashed line represents the line of equality in each plot. The red plus (+) in A-F indicates the median SNR of the two compared conditions. Pvalues from WSRT of the SNR or RT comparisons. $\boldsymbol{M}$ and $\boldsymbol{N}$ summarized the relationship between SNR, RT and congruency for two monkeys. 
The SNR differences in FEF reflect a neuronal congruency effect that can be partially dissociated from selective attention and/or certainty as indicated by RT

Next, we investigated whether the observed SNR differences can be explained by varying degrees of attentional deployment in congruent and incongruent conditions, since selective attention can also increase the SNR by enhancing the responses of task-relevant neurons and suppressing the responses of task-irrelevant neurons ${ }^{49-51}$. For example, it can be argued that subjects are more certain about the target location in congruent compared to incongruent conditions, which leads to more focused attention on the target (Figure 1B). In an attempt to investigate how attention affects SNR in the FEF between congruent and incongruent conditions in our experiment, we separated fast from slow trials for each condition based on the median RT of each condition for each recording session. Based on prior studies, we assume that subjects are more certain about the target location and/or attention is more focused on the target in fast compared to slow trials ${ }^{40-44}$. As expected, FEF neurons of both monkeys showed higher SNR in fast than slow trials in both congruent (Monkey S: Figure S4A, median SNR: 1.64 vs 1.40, $\mathrm{p}=$ 1.3e-5; Monkey R: Figure S4C, 1.23 vs 1.19, p = 0.066) and incongruent (Monkey S: Figure S4B, median SNR: 1.28 vs 1.08, p = 6.9e-10; Monkey R: Figure S4D, 1.10 vs 1.00, p = 8.6e-6) conditions, indicating that more focused attention indeed improves SNR within FEF. More importantly, we compared RT and SNR of congruent and incongruent conditions in fast and slow trials respectively.

In both monkeys, we found higher SNR in the FEF in congruent than incongruent conditions during both fast (Monkey S: Figure 5A, median SNR: 1.64 vs 1.28, p = 2e-9; Monkey R: Figure 5D, 1.23 vs 1.10, $\mathrm{p}=3.8 \mathrm{e}-6$ ) and slow (Monkey S: Figure 5B, median SNR: 1.40 vs 1.08, $\mathrm{p}=9.9 \mathrm{e}-11$; Monkey R: Figure 5E, 1.19 vs $1.00, \mathrm{p}=6.4 \mathrm{e}-10)$ trials. However, we only 
found significantly faster RTs to congruent than incongruent conditions in slow trials (Monkey S: Figure 5H, mean RT: 378 vs 388 ms, p =0.00049; Monkey R: Figure 5K, 390 vs 414 ms, p = 0.00029), but not in fast trials (Monkey S: Figure 5G, mean RT: 320 vs $318 \mathrm{~ms}, \mathrm{p}=0.30$; Monkey R: Figure 5J, 340 vs $340 \mathrm{~ms}, \mathrm{p}=0.55$ ). Secondly, we found that the RT in fast incongruent trials was much shorter than the slow congruent trials for both monkeys (Monkey S: Figure 5I, mean RT: 318 vs 378 ms, p = 0.00049; Monkey R: Figure 5L, 340 vs 390 ms, p= 0.00029), suggesting that certainty was higher and/or attention was actually more focused on targets during fast incongruent than slow congruent trials. Surprisingly, however, SNR in fast incongruent conditions was significantly lower than in slow congruent trials for both monkeys (Monkey S: Figure 5C, median SNR: 1.28 vs 1.40, $\mathrm{p}=0.00035$; Monkey R: Figure 5F, 1.10 vs $1.19, \mathrm{p}=3.9 \mathrm{e}-5)$

In sum, our results suggest that the SNR differences in FEF between congruent and incongruent conditions in our paradigm are modulated by both selective attention and congruency, but that congruency is a dominantly contributing factor (Figure 1B bottom, Monkey S: Figure 6M; Monkey R: Figure 6N). Therefore, we propose that a neuronal congruency signal is an additional independent factor besides selective attention that affects target selection.

\section{DISCUSSION}

Using a new task-switching paradigm, we found a behavioral congruency effect that can be linked to a neuronal congruency effect in FEF. The neuronal congruency effect comprises a signal change in task-relevant neurons and a noise change in task-irrelevant neurons, and their combination determines an SNR change within FEF's saliency map. The neuronal congruency 
meaning) cues the animal. Our results show that SNR changes in the FEF can be linked to dissociable congruency and selective attention mechanisms.

Previous single unit studies in monkey frontal and parietal areas showed that, depending on the specific experimental conflict paradigm, individual neurons can respond either higher or lower during incongruent versus congruent trials. Therefore, these neurons were proposed to be involved in either detecting or resolving conflicting information ${ }^{5,11,27,52-54}$. However, these studies did not consider the neurons' visual RF location. We showed that the RF positions relative to target locations are crucial to explain neuronal congruency effects, at least when spatial features are important and/or the recorded neurons have a visual RF as in our paradigm. Neuronal response differences between congruent and incongruent conditions in previous studies $^{5,11,27,52-54}$ can be explained by the relative amplitude between signal (carried by tasktask.

Human fMRI and EEG studies described higher responses in frontal cortex during higher conflict (incongruent) compared to lower conflict (congruent or neutral) conditions ${ }^{5,13,14,55}$. Due to the low spatial resolution of these techniques, it is impossible to distinguish task-relevant from 
task-irrelevant neurons in case they are homogeneously distributed within an area. Therefore, such results reflect an average population effect caused by congruency. For example, fMRI studies showed that activity in brain areas processing task-relevant features was amplified in high conflict/incongruent compared to low conflict/congruent conditions ${ }^{1,56}$. The higher responses in incongruent conditions can be explained if the amplitude of the changes in noise is larger than the signal changes at populational level, and/or if more neurons show noise change than signal changes. Both predictions are confirmed by our and previous studies: FEF neurons showed stronger noise than signal changes, indicated by the median absolute modulation indices (Figure S5). Moreover, more neurons showed significant noise than signal changes $(47 \%$ $(116 / 248)$ versus $33 \%(81 / 248)$, Chi-squared test, $\left.\chi^{2}=10.3, \mathrm{p}=0.001\right)$. Also, another study revealed that more neurons showed a higher response during incongruent than congruent trials in the $\mathrm{SEF}^{53}$. Thus, single-unit results predict an overall increased response during incongruent compared to congruent trials in fMRI and EEG studies.

In the current study, we analyzed the neuronal responses immediately before target dimming (i.e. $500 \mathrm{~ms}$ prior the go-cue for the behavioral response), long after the conflictwere matched between congruent and incongruent conditions during that time interval, leaving congruency as the major variable. In the trials we analyzed, the monkeys most likely focused their attention to the target well before target dimming because: i) the target location was determined by the rule- and color-cue in each trial, ii) there was a long delay between the colorcue and target dimming, which provided ample time to locate the target location, and iii) only correct trials were used in the analysis. Nevertheless, one may still argue that subjects are more certain about the target location in the congruent trials and/or that attention was more focused on 
the target compared to incongruent trials. Consequently, the SNR within FEF would be higher in congruent conditions $\mathrm{s}^{40,41,57}$. To investigate this possibility, we divided trials into slow and fast trials based on the median RT for each condition shown in Figure 3. Then we calculated the SNR to congruent and incongruent conditions for fast and slow trials. We can reasonably assume that the subjects were more certain about the target location and/or focused more attention on the target in fast than slow trials $\mathrm{s}^{40,41}$. If the SNR would be dominated by certainty and/or attention (indicated by RT), we would have expected higher SNR in fast compared to slow trials (Figure 1B top). On the other hand, if SNR is dominated by congruency, we would have expected higher SNR in congruent than incongruent trials (Figure 1B bottom). Our data matched the latter hypothesis. This suggests that SNR differences within the FEF are dominated by congruency levels in our experiment, although they are also sensitive to attention (Figure 6M, 6N).

The neuronal congruency effects observed in the FEF support connectionist models such as the parallel distributed processing (PDP) $\operatorname{model}^{12,58,59}$, which suggest that the interference from task-irrelevant information occurred automatically and was induced by prior training ${ }^{29,59}$. Such mechanisms may be generalized to other conflict paradigms (also without spatial components as in the present task). For example, trials with the word RED printed in red during the Stroop task are congruent trials, while BLUE trials printed in red are incongruent trials. Reporting red would be correct in both trial types. Given our results, we predict that 'red-coding' (task-relevant) neurons will respond higher, while 'blue-coding' (task-irrelevant) neurons will respond lower in congruent trials, and vice versa in the latter incongruent trials. The same rationale can be generalized to most if not all conflict-related paradigms. Where the neuronal congruency signals emerge within hierarchical processing streams, however, remains to be investigated in future studies. 


\section{References:}

1. Egner, T. \& Hirsch, J. Cognitive control mechanisms resolve conflict through cortical amplification of task-relevant information. Nat. Neurosci. 8, 1784-1790 (2005).

2. Miller, E. K. The prefontral cortex and cognitive control. Nat. Rev. Neurosci. 1, 59-65 (2000).

3. Miller, E. K. \& Cohen, J. D. An integrative theory of prefrontal cortex function. Annu. Rev. Neurosci. 24, 167-202 (2001).

4. Shinn-Cunningham, B. G. Object-based auditory and visual attention. Trends Cogn. Sci. 12, 182-186 (2008).

5. Mansouri, F. A., Egner, T. \& Buckley, M. J. Monitoring Demands for Executive Control: Shared Functions between Human and Nonhuman Primates. Trends Neurosci. 40, 15-27 (2017).

6. Mansouri, F. A., Tanaka, K. \& Buckley, M. J. Conflict-induced behavioural adjustment: A clue to the executive functions of the prefrontal cortex. Nat. Rev. Neurosci. 10, 141-152 (2009).

7. Ito, S., Stuphorn, V., Brown, J. W. \& Schall, J. D. Performance Monitoring by the Anterior Cingulate Cortex During Saccade Countermanding. Science (80-. ). 302, 120 122 (2003).

8. Stroop, J. R. Studies of interference in serial verbal reactions. J. Exp. Psychol. 18, 643662 (1935).

9. Eriksen, B. A. \& Eriksen, C. W. Effects of noise letters upon the identification of a target letter in a nonsearch task. Percept. Psychophys. 16, 143-149 (1974).

10. Hommel, B. Psychologische Forschung The role of attention for the Simon effect. Psychol. Res. 55, 208-222 (1993).

11. Munoz, D. P. \& Everling, S. Look away: The anti-saccade task and the voluntary control of eye movement. Nat. Rev. Neurosci. 5, 218-228 (2004).

12. MacLeod, C. M. Half a century of research on the Stroop effect: An integrative review. Psychol. Bull. 109, 163-203 (1991).

13. Egner, T. Congruency sequence effects and cognitive control. Cogn. Affect. Behav. Neurosci. 7, 380-390 (2007).

14. Banich, M. T. The Stroop Effect Occurs at Multiple Points Along a Cascade of Control: Evidence From Cognitive Neuroscience Approaches. Front. Psychol. 10, 2164 (2019).

15. Stoet, G. \& Snyder, L. H. Neural correlates of executive control functions in the monkey. Trends Cogn. Sci. 13, 228-234 (2009).

16. Cespón, J., Hommel, B., Korsch, M. \& Galashan, D. The neurocognitive underpinnings of the Simon effect: An integrative review of current research. Cognitive, Affective and Behavioral Neuroscience 1-17 (2020). doi:10.3758/s13415-020-00836-y

17. Sheth, S. A. et al. Human dorsal anterior cingulate cortex neurons mediate ongoing 
behavioural adaptation. Nature 488, 218-221 (2012).

18. Smith, E. H. et al. Widespread temporal coding of cognitive control in the human prefrontal cortex. Nat. Neurosci. 22, 1883-1891 (2019).

19. Bardi, L., Kanai, R., Mapelli, D. \& Walsh, V. TMS of the FEF Interferes with Spatial Conflict. J. Cogn. Neurosci. 24, 1305-1313 (2012).

20. Nuiten, S. A. et al. Preserved sensory processing but hampered conflict detection when stimulus input is task-irrelevant. Elife 10, e64431 (2021).

21. Coulthard, E. J., Nachev, P. \& Husain, M. Control over Conflict during Movement Preparation: Role of Posterior Parietal Cortex. Neuron 58, 144-157 (2008).

22. Parris, B. A. et al. An fMRI study of response and semantic conflict in the stroop task. Front. Psychol. 10, 2426 (2019).

23. Taylor, S. F., Kornblum, S., Lauber, E. J., Minoshima, S. \& Koeppe, R. A. Isolation of specific interference processing in the stroop task: PET activation studies. Neuroimage 6, 81-92 (1997).

24. Botvinick, M., Nystrom, L. E., Fissell, K., Carter, C. S. \& Cohen, J. D. Conflict monitoring versus selection for-action in anterior cingulate cortex. Nature 402, 179-181 (1999).

25. Banich, M. T. et al. fMRI studies of stroop tasks reveal unique roles of anterior and posterior brain systems in attentional selection. J. Cogn. Neurosci. 12, 988-1000 (2000).

26. Casey, B. J. et al. Dissociation of response conflict, attentional selection, and expectancy with functional magnetic resonance imaging. Proc. Natl. Acad. Sci. U. S. A. 97, 8728-33 (2000).

27. Nakamura, K., Roesch, M. R. \& Olson, C. R. Neuronal Activity in Macaque SEF and ACC During Performance of Tasks Involving Conflict. J. Neurophysiol. 93, 884-908 (2005).

28. Egner, T. \& Hirsch, J. The neural correlates and functional integration of cognitive control in a Stroop task. Neuroimage 24, 539-547 (2005).

29. MacLeod, C. M. Attention: Beyond Stroop's (1935) colour-word interference phenomenon. in Cognitive Psychology: Revisiting the Classic Studies (eds. Eysenck, M. . \& Groome, D.) 60-70 (SAGE Publications, 2015).

30. Algom, D. \& Chajut, E. Reclaiming the Stroop effect back from control to input-driven attention and perception. Front. Psychol. 10, 1683 (2019).

31. Schmidt, J. R. Evidence against conflict monitoring and adaptation: An updated review. Psychon. Bull. Rev. 26, 753-771 (2019).

32. Melara, R. D. \& Algom, D. Driven by Information: A Tectonic Theory of Stroop Effects. Psychol. Rev. 110, 422-471 (2003).

33. Botvinick, M. M., Braver, T. S., Barch, D. M., Carter, C. S. \& Cohen, J. D. Conflict monitoring and cognitive control. Psychol. Rev. 108, 624-652 (2001).

34. Thompson, K. G. \& Bichot, N. P. A visual salience map in the primate frontal eye field. 
Prog. Brain Res. 147, 251-262 (2005).

35. Sato, T., Murthy, A., Thompson, K. G. \& Schall, J. D. Search efficiency but not response interference affects visual selection in frontal eye field. Neuron 30, 583-591 (2001).

36. Egner, T. Multiple conflict-driven control mechanisms in the human brain. Trends Cogn. Sci. 12, 374-380 (2008).

37. MacLeod, C. M. The Stroop task: The 'gold standard' of attentional measures. J. Exp. Psychol. Gen. 121, 12-14 (1992).

38. Maunsell, J. H. R. \& Treue, S. Feature-based attention in visual cortex. Trends Neurosci. 29, 317-322 (2006).

39. Maunsell, J. H. R. Neuronal Mechanisms of Visual Attention. Annu. Rev. Vis. Sci. 1, 373391 (2015).

40. Kiani, R., Corthell, L. \& Shadlen, M. N. Choice certainty is informed by both evidence and decision time. Neuron 84, 1329-1342 (2014).

41. Kiani, R. \& Shadlen, M. N. Representation of confidence associated with a decision by neurons in the parietal cortex. Science (80-. ). 324, 759-764 (2009).

42. Cohen, M. R. \& Maunsell, J. H. R. Attention improves performance primarily by reducing interneuronal correlations. Nat. Neurosci. 20091212 12, 1594-1600 (2009).

43. Carrasco, M. Visual attention: The past 25 years. Vision Res. 51, 1484-1525 (2011).

44. Posner, M. I., Snyder, C. R. R. \& Davidson, B. J. Attention and the Detection of Signals. J. Exp. Psychol. Gen. 109, 160-174 (1980).

45. Corbetta, M. \& Shulman, G. L. Control of goal-directed and stimulus-driven attention in the brain. Nat. Rev. Neurosci. 3, 201-215 (2002).

46. Schall, J. D. The neural selection and control of saccades by the frontal eye field. Philos. Trans. R. Soc. Lond. B. Biol. Sci. 357, 1073-82 (2002).

47. Wardak, C., Ibos, G., Duhamel, J. R. \& Olivier, E. Contribution of the monkey frontal eye field to covert visual attention. J. Neurosci. 26, 4228-4235 (2006).

48. Goodale, M. A. \& Milner, A. D. Separate visual pathways for perception and action. Trends Neurosci. 15, 20-5 (1992).

49. Thompson, K. G., Biscoe, K. L. \& Sato, T. R. Neuronal basis of covert spatial attention in the frontal eye field. J. Neurosci. 25, 9479-9487 (2005).

50. Joiner, W. M., Cavanaugh, J., Wurtz, R. H. \& Cumming, B. G. Visual responses in FEF, unlike V1, primarily reflect when the visual context renders a receptive field salient. $J$. Neurosci. 37, 9871-9879 (2017).

51. Sato, T., Thompson, K., Schall, J., Murthy, A. \& Bichot, N. Visual Processing in the Macaque Frontal Eye Field. in The Primate Visual System (eds. Kaas, J. H. \& Collins, C.) 205-230 (CRC press, 2003). doi:10.1201/9780203507599.ch9

52. Mansouri, F. A., Buckley, M. J. \& Tanaka, K. Mnemonic function of the dorsolateral prefrontal cortex in conflict-induced behavioral adjustment. Science (80-. ). 318, 987-990 (2007). 
53. Olson, C. R. \& Gettner, S. N. Neuronal activity related to rule and conflict in macaque supplementary eye field. Physiol. Behav. 77, 663-670 (2002).

54. Merriam, E. P. et al. Stimulus-response incompatibility activates cortex proximate to three eye fields. Neuroimage 13, 794-800 (2001).

55. Janssens, C., De Loof, E., Boehler, C. N., Pourtois, G. \& Verguts, T. Occipital alpha power reveals fast attentional inhibition of incongruent distractors. Psychophysiology 55, 1-11 (2018).

56. Polk, T. A., Drake, R. M., Jonides, J. J., Smith, M. R. \& Smith, E. E. Attention enhances the neural processing of relevant features and suppresses the processing of irrelevant features in humans: A functional magnetic resonance imaging study of the stroop task. $J$. Neurosci. 28, 13786-13792 (2008).

57. Parr, T. \& Friston, K. J. Attention or salience? Curr. Opin. Psychol. 29, 1-5 (2019).

58. Musslick, S. \& Cohen, J. D. Rationalizing constraints on the capacity for cognitive control. Trends Cogn. Sci. 25, 757-775 (2021).

59. Cohen, J. D., Dunbar, K. \& McClelland, J. L. On the control of automatic processes: A parallel distributed processing account of the stroop effect. Psychol. Rev. 97, 332-361 (1990). 


\section{Methods}

2

\section{Subjects and setup}

Two adult male rhesus monkeys (Macaca mulatta, 6-8.5 kg, 8 and 10 years old during the period of recordings, respectively) participated in the current study. All experimental procedures and animal care were performed in accordance with the National Institute of Health's Guide for the care and use of laboratory animals, European legislation (Directive 2010/63/EU) and were approved by the Ethical Committee of KU Leuven. The animals were socially group-housed in cages between 16 and $32 \mathrm{~m}^{3}$ equipped with enrichment devices (toys, woods, ropes, foraging devices etc.) at the primate facility of the KU Leuven Medical School. The animals were exposed to natural light and additional artificial light for $12 \mathrm{~h}$ every day. During the study, the animals had unrestricted access to food and daily access to restricted volumes of fruits and water. On training and experimental days, the animals were allowed unlimited access to fluid through their performance during the experiments. Using operant conditioning techniques with positive reinforcers, the animals received fluid rewards for every correctly performed trial. Throughout the study, the animals' psychological and veterinary welfare was monitored daily by the veterinarians, the animal facility staff and the lab's scientists, all specialized in working with non-human primates. The two animals were healthy at the conclusion of our study and were subsequently employed in other studies.

Each monkey was implanted with an MRI-compatible head holder to minimize head movements during the training and recording. One standard recording chamber was also implanted in each monkey above the right frontal cortex to allow access to FEF, with implantation locations chosen based on preceding MRI scans. The details of the implant surgery was previously described in ${ }^{1}$. 
The experiments were performed in a dimly lit room with the only source of light being the display monitor. A Dell 17 inches LCD monitor at a distance of $57 \mathrm{~cm}$ from the monkeys' eyes was used to display the visual stimulus at a refresh rate of $60 \mathrm{~Hz}$ and a spatial resolution of around 40 pixels per degree. The monkeys were seated in a sphinx position in a custom-made primate chair, typically used for fMRI experiments 1. Stimulus presentation, reward delivery, 6 electrophysiological and behavioral data collection was controlled by custom software controlled 7 by custom-built hardware and Dell Windows computers. The exact timing of the stimulus onsets 8 and offsets was monitored by a photocell attached to the bottom-right corner of the LCD monitor. 9 Eye-positions were monitored by an Iscan (Iscan, MA, USA) Infrared corneal reflection system at $10120 \mathrm{~Hz}$.

11 Neuronal activity was recorded extracellularly with Plexon 16-chanel V-probes (Plexon Inc., TX, USA). The 16 recording sites were aligned in a row with 150 um inter-site spacing. The neuronal signal was filtered $(300-1000 \mathrm{~Hz})$, amplified, digitized, and stored with a TDT system

14 (TDT Inc., TX, USA) with a $23 \mathrm{kHz}$ sampling rate. All neuronal signals were recorded and stored for offline analyses. Offline spike sorting was performed with Plexon's Offline Sorter to isolate single and multi units. The FEF was identified by referencing the recordings to the structural MRI, in addition to the functional properties of the recorded neurons. Structurally, the recording sites, in the anterior bank of the arcuate sulcus, were localized with T1-weighted MRI imaging (TR = $2.5 \mathrm{~s}, \mathrm{TE}=4.35 \mathrm{~ms}, \mathrm{TI}=850 \mathrm{~ms}$ ) (Figure S1). Functionally, the saccade direction and spatial tuning of the neurons was visually inspected online. A site was considered to be within the FEF, 21 only when the neurons from at least 1 channel showed clear direction tuning for saccades and spatial tuning for visual stimuli (in all our recording sessions included in current study, we actually observed that the neurons from multiple channels showed tuning to spatial location and saccade 
1 directions). By combining the structural and functional evidence, we are confident that the locations we recorded from were in FEF.

\section{Behavioral Tasks and stimuli}

Once the recording 16-channel-probe (Plexon V-probe) arrived at target depth and the neuronal signals were stabilized, the monkeys first performed a fixation task whereby they maintained fixation on a central black fixation point ( 0.2 by 0.2 degrees). We identified the RF of neurons recorded from several channels by briefly flashing $(200 \mathrm{~ms})$ a white square stimulus on the gray background at one of 25 locations ( 5 by 5 grid, covering 25 by 20 degrees of the visual field). The RF location was determined by online inspection and analysis of the neuronal responses to the flashed stimuli. We could not map the RFs for the neurons recorded at all channels, since we focused on just a few channels during the recording, and the amount of trials that the subjects could perform was limited per day (preventing us to carefully map all the RFs from all neurons on all channels). After mapping the RFs from several channels, we selected that location covering most of the RFs based on the mapping results, for placing the target and distractor during the main task (Figure 2). Next, we measured the neuron's saccade direction tuning by asking the monkeys to perform a visually guided saccade task from the center fixation point to a peripheral saccade target ( 7 visual degrees from the center fixation point). The saccade target was randomly picked from a set of 8 possible locations (evenly separated by $45^{\circ}$ around a circle). We visually inspected the saccade direction tuning online. After identification of the location to position the stimuli, and part of the neurons showed tuning for saccade directions, we switched to the main task and recorded neuronal activity without further moving the V-probe (Figure 2). 
For the main experiment, the monkeys were trained to perform a task-switching paradigm

2 (Figure 2), where they were trained to pay covert attention to a target stimulus and respond to its

3 dimming while ignoring a distractor stimulus. The monkeys initiated a trial by foveating a black

4 fixation point ( 0.2 by 0.2 degrees) at the center of the screen. After $500 \mathrm{~ms}$ of fixation, the fixation

5 point changed to a horizontal ( 0.4 by 0.2 degrees) or vertical ( 0.2 by 0.4 degrees) fixation bar

6 which served as task rule-cue (color rule, or spatial rule, respectively) for the current trial.

7 Accordingly, for a horizontal bar (color rule), the target stimulus was indicated by the color of the

8 subsequently shown color-cue (which appeared $1100 \mathrm{~ms}$ after the task rule-cue, e.g., red or cyan

9 for left, and pink or blue for right; please note that we used two pairs of color-cues (red-pink and

10 cyan-blue) for monkey R (each recording session only used one pair), and only one pair (red-pink)

11 was used for Monkey S). The spatial location of the color-cue was irrelevant in these trials.

12 Alternatively, when a vertical bar appeared (spatial rule), the target stimulus was indicated by the

13 spatial location of the subsequent color-cue, its color being irrelevant. The rule-cue was presented

14 for $500 \mathrm{~ms}$, then, the original squared fixation point returned together with a pair of white peripheral

15 stimuli (1 by 1 degree). The two stimuli were positioned at equal eccentricities, one of them was

16 presented at the location determined by the RF mapping task within most of the recorded neurons'

17 RFs, the other one at 180 degrees from the former (thus the two stimuli were central symmetrical,

18 if one stimulus was presented in the top left quadrant, the second appeared in the bottom right

19 quadrant). This alignment would maximize the distance between the two stimuli and ensure that

20 one of them was within the recorded neurons' RF, while the other one was out (in present study,

21 the two stimuli were separated by at least 14 visual degrees). After a delay of $600 \mathrm{~ms}$ following

22 stimuli onset, one of the two white squares turned into a color-cue ( 1 by 1 degree) for $200 \mathrm{~ms}$.

23 Combined with the rule-cue, the target location was indicated either by the color (color-rule: 
horizontal bar; red and pink indicated that the target would be located at the left and right, respectively), or the location of the color-cue independent of its color (spatial rule: vertical bar).

The monkeys had to respond to a brief $(150 \mathrm{~ms})$ dimming in luminance of the target by pressing a button with their left hand (within 200-700ms after the dimming). Target dimming occurred between 660-1950ms after color-cue offset in every trial. Moreover, to ensure that the subjects were attending to the target rather than responding to any dimming, the subjects had to ignore similar dimming of the distractor, which happened randomly in $50 \%$ of the trials, and never more than once in a trial. Distractor dimming occurred between 200-1500ms after color-cue offset, with the additional requirement that it happened at least $300 \mathrm{~ms}$ before target dimming. This separation ensured that the monkeys' responses to the distractor dimming could be identified and distinguished from their responses to the target dimming. Trials terminated 700ms after the target dimming, and the monkey received a drop of juice if the button had been correctly pressed during this period. Note that there was a target dimming in each trial, so the monkey was required to make an identical operant response in each trial in order to be rewarded, thus we excluded a stimulus-response conflict. During the task, the background was always gray (RGB values: 70, 70, $70 ; 4 \mathrm{~cd} / \mathrm{m}^{2}$ ); the fixation point and the task rule-cue (horizontal and vertical fixation point) were black (RGB: $0,0,0 ; 0.1 \mathrm{~cd} / \mathrm{m}^{2}$ ); the squared stimuli were white (RGB: 255, 255, 255; $77 \mathrm{~cd} / \mathrm{m}^{2}$ ); the dimming of the squared stimuli corresponded to a gray stimulus (RGB: 210, 210, 210; 51 $\mathrm{cd} / \mathrm{m}^{2}$ ). Monkeys had to maintain fixation within a (virtual) squared window of 2.5-3 visual degrees centered around the fixation point until they received the reward. Please note that in the paradigm, between the congruent and incongruent conditions, we controlled 1) the visual input of the stimuli, by analyzing the neuronal response to exactly the same stimuli in the delay period; 2)the response of the subjects by asking the subjects performing the same response in all trials; 3 ) 
1 the allocation of spatial attention by requiring subjects to attend the target stimulus within or out 2 of the neurons' RF.

\section{Data analysis}

The behavioral and neuronal data analysis was performed using MATLAB (MathWorks, MA, USA). We performed 12 recording sessions in Monkey S, and 17 recording sessions in Monkey $\mathrm{R}$, and all the sessions included 32-40 correct trials for each condition shown in Figure 3. The correct trials (hits) corresponded to trials in which the button was pressed within $200-700 \mathrm{~ms}$ after target dimming. Incorrect trials included all false alarm trials (i.e., when the monkeys pressed the button at the wrong time) and missed trials (the monkeys did not press a button within the 200-

$11700 \mathrm{~ms}$ response window after target dimming). All trials during which fixation was interrupted were excluded from the analysis. The performance of each session was defined as the number of correct trials divided by the sum of the number of correct and incorrect trials.

Neuronal activity was recorded from the FEF in the right hemisphere using Plexon's 16channel V-probes. The spikes were offline sorted into single- and multi-units using Plexon's offline sorter. A total of 591single neurons (267 from Monkey S, 324 from Monkey R) were isolated using offline sorting. Since our design required that one of the two stimuli should be presented in the neurons' RF, and not all of the neurons satisfied with this since: 1) some of the neurons were not visually driven (by these white flashing squares) in FEF, 2) multiple neurons were recorded with the probe at the same time, some of their RFs did not cover the target nor the distractor. Therefore, we first identified the visually-driven neurons that were activated by the white squared stimuli before the color cues. A neuron was qualified as visually-driven when it 23 showed a significantly higher response in the $0-500 \mathrm{~ms}$ time window after the onset of the two 
1 stimuli onset compared to the response in 200-500ms after onset of the fixation point. (two tailed 2 paired t-test, $\mathrm{p}<0.05)$ in all correct trials. Next, for these visually-driven neurons, to determine their RF at the left or right visual hemifield, we analyzed the neuronal response induced by the target dimming. Since we recorded from the right FEF, we would expect most of the neurons' RFs were in the left visual hemifield ${ }^{2}$. We only included those neurons in our further analysis that showed a significantly higher response to the target dimming in the left compared to the right 7 hemifield $(50-200 \mathrm{~ms}$ after target dimming onset, two tailed paired t-test, $\mathrm{p}<0.05)$. Therefore, the 8 RFs of the selected neurons would cover targets presented in the left visual hemifield, while 9 targets presented in the right visual field were outside the neuron's RFs, which was confirmed by the neuronal response in figure $3 \mathrm{AB}$. We found a clear response to the target dimming when the target was supposed to be inside the RF (solid lines), while the response to the target dimming was not clear when the target was supposed to be out of the RF (dashed lines). Using the additional criteria, we were able to select 248 visual neurons with pronounced contralateral (left) RFs (121 from Monkey S, 127 from Monkey R) for further analysis.

Peri-stimulus time histograms (PSTHs) were calculated by smoothing the data with a Gaussian-weighted moving average over each $50 \mathrm{~ms}$ time bins in a window of $200 \mathrm{~ms}$. The average activity across trials was first calculated for each neuron, and then, to obtain the displayed PSTHs in Figure 3A and 3B, the PSTHs of individual neurons were averaged across all neurons. For the bin-by-bin statistical significance tests (Figure 3A and 3B), we performed a paired t-test across neurons for each given bin based on the non-smoothed average response of each neuron, if a test between two conditions was significant/non-significant for a given bin, we assumed that it was significant/non-significant for the entire duration of the $50 \mathrm{~ms}$ bin. To avoid that the transient response to the brief dimming of the distractor stimulus affected the results, for the trials including 
1 a distractor dimming, we excluded the period of $0-200 \mathrm{~ms}$ following the distractor dimming onset

2 from the PSTH and firing-rate calculations. For the differences between two conditions (Figure $3 \quad 3 \mathrm{C}-3 \mathrm{~F}$ ) at neuron level, we reported the modulation effects using a modulation index (MI) for each

4 neuron, which was defined as the difference in the firing-rates for the two conditions divided by

5 their sum for a time window of 500ms before target dimming onset. We used the median value of

6 the MIs of all neurons to report the population modulation effect, and used a WSRT to test whether

7 the effect was significant. The same analysis was performed for trials with a color-rule and a 8 spatial-rule (Figure S2-S4).

9 For the SNR analysis, we first divided all the trials in each recording session into fast and 10 slow trials based on the median RT for each condition shown in Figure 4, i.e., the fast and slow 11 trials for TarIn_con, TarIn_incon, TarOut_con, and TarOut_incon. The avarage neuronal response 12 of the $500 \mathrm{~ms}$ before target dimming to TarIn_con ( $\left.\mathrm{R}_{\text {TarIn_con }}\right)$ and TarIn_incon ( $\left.\mathrm{R}_{\text {TarIn_incon }}\right)$ 13 represented the 'signal' in congruent and incongruent trials respectively, while the $\mathrm{R}_{\text {TarOut_con }}$ and $14 \mathrm{R}_{\text {TarOut_incon }}$ represented the 'noise'. Then, the SNR of the FEF neurons for the congruent and 15 incongurent trials was calculated as $\mathrm{R}_{\text {TarIn_con }} / \mathrm{R}_{\text {TarOut_con }}$ and $\mathrm{R}_{\text {TarIn_incon }} / \mathrm{R}_{\text {TarOut_incon }}$ respectively for 16 each recording session. For each neruon, we had the SNR for 4 conditions, i.e., fast_congruent 17 condition, fast_incongurent condition, slow_congruent condition, slow_incongurent condition. 18 We compared the SNR differential based on these 4 conditions to investigate the relationship 19 between congurency effect and top-down modulations (Figure 6). 


\section{References:}

2

3 1. Vanduffel, W. et al. Visual Motion Processing Investigated Using Contrast Agent-

$4 \quad$ Enhanced fMRI in Awake Behaving Monkeys. Neuron 32, 565-577 (2001).

5 2. Patrick Mayo, J., DiTomasso, A. R., Sommer, M. A. \& Smith, M. A. Dynamics of visual

6 receptive fields in the macaque frontal eye field. J. Neurophysiol. 114, 3201-3210 (2015). 


\section{ACKNOWLEDGMENTS}

The authors thank C. Fransen, A. Coeman, P. Kayenbergh, I. Puttemans, C. Ulens, A. Hermans,

G. Meulemans, M. Depaep, W. Depuydt and S. Verstraeten for technical and administrative support. We thank T. Verguts for his comments on the manuscript. This work received funding from KU Leuven C14/17/109, IDN/20/016; Fonds Wetenschappelijk Onderzoek-Vlaanderen (FWO-Flanders) G0B8617N, G094816N, G0E0520N, G0C1920N, 1501320N, 12W0919N; the European Union's Horizon 2020 Framework Programme for Research and Innovation under Grant Agreement No 945539 (Human Brain Project SGA3). T.Y is postdoctoral fellow of the FWO-Flanders.

Author contributions: Conceptualization, T.Y, and W.V., Methodology, T.Y, and W.V., Investigation, T.Y, Writing, T.Y, and W.V., Funding acquisition, T.Y, and W.V., Supervision, W.V.

Data and Materials will be made available through EBRAINS.

The authors declare no competing interests. 


\section{Supplementary Files}

This is a list of supplementary files associated with this preprint. Click to download.

- YaoSMfiguresNCfinal.docx 